\title{
¿Por qué no editan en la Wikipedia los alumnos universitarios?
}

\section{Why Don't University Students Edit on Wikipedia?}

Ángel Obregón-Sierra

Universidad Isabel I (España)

Natalia González-Fernández

Universidad de Cantabria (España)
Los alumnos universitarios admiten que una de las herramientas que más utilizan en su vida diaria es la Wikipedia, una enciclopedia en línea que da libertad para que cualquier persona pueda editar su contenido. Consideran que esta es fiable y útil para sus consultas, pero sobre todo destacan que contiene datos actuales. A pesar de estos aspectos positivos, se considera que menos del 1\% de los alumnos edita frecuentemente en ella para mejorar sus contenidos. Para conocer los motivos por los cuales no editan en ella, en esta investigación hemos implementado el método mixto secuencial, para obtener información de tres herramientas de obtención de datos como son los cuestionarios, los grupos de discusión y las entrevistas. Una vez analizadas las 1173 respuestas de los cuestionarios, realizamos un grupo de discusión con los alumnos de la Universidad de Barcelona y entrevistas a expertos editores en la Wikipedia, y se constató que solo el 0,38\% edita frecuentemente o muy a
College students admit that one of the tools they use the most in their daily lives is Wikipedia, an online encyclopedia that gives anyone the freedom to edit its content. They consider that it is reliable and useful for their queries, but above all they believe that it contains current data. In spite of these positive aspects, it is considered that less than $1 \%$ of the students frequently edit it to improve its contents. In order to determine the reasons why they do not edit it, in this research we have implemented the sequential mixed method to obtain information from three data collection tools such as questionnaires, discussion groups and interviews. Once the 1,173 responses to the questionnaires had been analyzed, we carried out a discussion group with students from the University of Barcelona and interviews with expert editors on Wikipedia, and it was found that only $0.38 \%$ edited frequently or very often. Several reasons were given for not editing it, but mainly the students indica- 
menudo. Se expusieron varios motivos para no editar en ella, pero principalmente los alumnos indicaron que no se sentían capaces de aportar ningún tipo de información.

Palabras clave: Wikipedia, Wikis, Web 2.0, estudiantes universitarios, trabajo colaborativo. ted that they did not feel capable of providing any type of information.

Key words: Wikipedia, Wikis, Web 2.0, university students, collaborative work.

a Wikipedia es una enciclopedia en línea, editada por voluntarios de todo el mundo en más de 300 idiomas, uno de los cuales es la versión en español, que suele encontrarse entre el tercer y el quinto idioma más visitado. Además, es la segunda en número de usuarios registrados, muy por delante del resto de versiones. Es el cuarto idioma en cuanto a usuarios activos (los que realizan más de 100 ediciones al mes), pero a pesar de estos números, solo es la novena con mayor número de artículos a comienzos de 2020, más de millón y medio (Zachte, 2020).

Según los números expuestos, parece que muchos usuarios se registran en la versión en español, pero finalmente no contribuyen, como sí hacen en otros idiomas. Cada año se realizan más proyectos educativos en los que se utiliza la Wikipedia, enseñando a los alumnos su funcionamiento para que la utilicen correctamente. Estos proyectos ayudan a aumentar el número de usuarios registrados, pero al no continuar editando no aumentan ni los usuarios activos ni el número de artículos creados.

Head y Eisenberg (2009) realizaron una investigación sobre el uso de la Wikipedia por los estudiantes universitarios de varias instituciones estadounidenses. Constataron que más del 50\% de los alumnos universitarios estadounidenses consultaba la Wikipedia con asiduidad y más del 75\% lo hacía en ocasiones, aunque el porcentaje de editores era muy bajo. El 1\% admitía editar frecuentemente, el 20\% lo hacía rara vez, mientras que el 77\% no lo había hecho nunca. En otras investigaciones (Kleimann et al., 2008), el número de editores era similar, el 0,3\% creaba artículos con frecuencia, mientras que el $83 \%$ nunca lo había hecho.

Otras investigaciones (Flanagin y Metzger, 2011) han constatado que la mayoría de los alumnos encuestados conocía la Wikipedia, aunque el 22\% no sabía que todo el mundo podía participar en ella. Sahut et al. (2015) han afirmado que solo la quinta parte de los alumnos ha recibido explicaciones del funcionamiento de la Wikipedia, y de ellos, más del 30\% fueron asistidos por gente cercana, pero no por profesores, lo que puede ocasionar problemas si dichas personas no disponen de conocimientos profundos en la temática. En la misma línea encontramos las investigaciones de Forte y Bruckman (2010), que constatan que los alumnos 
no han sido formados debidamente y que por ese motivo ven una complejidad añadida al hecho de aportar citas a sus trabajos o editar en la Wikipedia. Los profesores suelen tener una actitud pasiva en el proceso de enseñar a los alumnos a utilizarla (Meishar-Tal, 2015).

A pesar de ello, en los últimos años la Wikipedia ha sido utilizada como soporte para las actividades escolares y universitarias (Fessakis y Zoumpatianou, 2012; Menchen-Trevino y Hargittai, 2011). En Francia, el primer acercamiento educativo con la Wikipedia fue realizado por la Universidad de París VIII en el marco de un proyecto que se denominó P8 y que duró cinco años consecutivos. Después se han realizado otros proyectos como Egypte, de Mario Asselin, y recientemente el Wikiconcurso, de Gilles (2015).

En la Wikipedia en inglés los equipos precursores fueron Kevin Zollman en la Universidad de Indiana y Andrew Lin en la de Hong Kong (Endrizzi, 2006). Unos años más tarde, en 2010, se puso en marcha una iniciativa en Estados Unidos para fomentar la incorporación de la Wikipedia en varios niveles educativos, para lo cual se crearon los embajadores, usuarios de la Wikipedia que colaboraban con el profesorado (Saorín, 2012). A comienzos de 2014 se anunció que, desde la Universidad de Berkeley, Estados Unidos, se había propuesto como objetivo formar a los estudiantes para que estos contribuyesen a la Wikipedia. Para ello se contrató al profesor Kevin Gorman, un geógrafo de 24 años gran conocedor de la Wikipedia (De Queiroz, 2014).

En la Universidad de Hope, en Liverpool, se realizó un estudio sobre el uso de la Wikipedia por profesores y alumnos (Knight y Pryke, 2012). Se basaba en los datos de 133 profesores y 1.222 alumnos, que indicaron que el $75 \%$ la usaba, y que una cuarta parte de los profesores guiaba a sus alumnos en cómo utilizarla, tratando de unirlo a sus enseñanzas. Las temáticas son cada vez más variadas, y en unos casos se enseñan definiciones, se escriben artículos, se realizan artículos destacados, traducciones, se agregan fotografías, ilustraciones o vídeos (Peters, 2012).

En España, la Universidad de Las Palmas y la de Murcia publican habitualmente contenidos en wikis y trabajan con la Wikipedia (Saorín et al., 2011). También son habituales las publicaciones realizadas desde la Universitat Oberta de Catalunya (Obregón, 2019), donde han observado que el uso activo de la Wikipedia fue positivo para los alumnos y mejoró su rendimiento académico (MeseguerArtola, 2014). Otras universidades que han incluido la Wikipedia en el entorno de trabajo de los estudiantes han sido la Complutense, la de Salamanca y la de Oviedo (Sánchez, 2014).

Por el contrario, en la utilización de la Wikipedia no todo son aspectos positivos. Algunas investigaciones han constatado un problema entre el mantenimiento de la calidad y la integración de nuevos colaboradores (Sahut y Chabriac, 2015). Durante un experimento realizado por Ebner, Zechner y Holzinger (2010), estos determinaron que sus alumnos no habían realizado apenas colaboraciones en un wiki creado como una enciclopedia, en su opinión, por tres motivos principales:

- Para los alumnos es inimaginable trabajar voluntariamente para el bien de la comunidad. 
16 - Habitualmente los editores aparecen después de un tiempo de lectura, y no inmediatamente, por lo que es necesario más tiempo para que exista un número considerable de editores.

- No existía durante todo el tiempo de uso una sensación de comunidad y de trabajo en equipo.

La problemática no es únicamente esta, sino que en algunos casos los alumnos universitarios no tienen suficiente preparación en temas tecnológicos o informáticos, porque en sus etapas anteriores no han sido trabajadas por sus profesores, lo que dificulta la compresión de algunos conceptos (Monereo, 2009). También se da el caso de la poca preparación de los profesores, que se sienten inseguros a la hora de desarrollar este tipo de herramientas, y pueden verse superados por los propios alumnos (Gómez et al., 2014).

\section{METODOLOGÍA}

Atendiendo a la realidad expuesta en la introducción, presentamos este trabajo, cuyos objetivos son: constatar el conocimiento que tienen los alumnos universitarios sobre la Wikipedia, conocer si editan o han editado en alguna ocasión esta enciclopedia en línea y, en caso de no hacerlo, obtener los motivos por los que no editan en ella, a pesar de utilizarla habitualmente para temas relacionados con el ocio y para sus estudios.

\section{INSTRUMENTO Y PROCEDIMIENTO}

Para dar respuesta a nuestros objetivos, en esta investigación se utilizó el método mixto secuencial, de manera que se realizó una mezcla entre los fundamentos teóricos descritos anteriormente y tres herramientas de obtención de datos como son los cuestionarios, los grupos de discusión y las entrevistas.

Tras realizar una revisión de la literatura científica sobre los proyectos educativos realizados con la Wikipedia, se diseñó un cuestionario ad hoc para recoger información cuantitativa. Antes de utilizarse, se realizó su validación gracias a la técnica Delphi y a la colaboración de diez profesores expertos para su verificación. Las propuestas de los expertos generaron la modificación de varias preguntas, la aclaración del texto de introducción y la eliminación y creación de ítems, para obtener un IVC de ,985 en el primer envío. Después de reenviar el cuestionario con las modificaciones propuestas, aumentó el IVC y se procedió a la realización de una prueba piloto con alumnos universitarios de Educación de la Universidad de Cantabria. Tras un tiempo de espera para su realización, se obtuvieron 73 respuestas. Estas se analizaron con el programa estadístico SPSS, que nos ayudó a perfeccionar más el cuestionario, ya que se eliminaron los ítems que no mostraban una buena adecuación muestral $(\mathrm{KMO}<, 5)$. Los ítems se agruparon en cuatro dimensiones tras realizar el Análisis Factorial y obtener un Alpha de Cronbach de ,878. Después de finalizar todas estas fases se eliminó un total de 23 ítems. 
Una vez completada la fase de elaboración del cuestionario se procedió a enviar correos electrónicos a todas las universidades de España con estudios sobre Educación, tanto públicas como privadas. Tras varias semanas de apertura del cuestionario, se obtuvieron 1.415 respuestas, antes de validarlas, ya que algunas podían haber sido cumplimentadas por profesores o estudiantes en otros niveles educativos. El resultado final, después de constatar las respuestas válidas, fue de 1.173 cuestionarios cumplimentados.

A continuación, se realizaron estudios descriptivos, de fiabilidad e inferenciales, como las pruebas de Kruskal-Wallis (para comprobar si existen diferencias significativas entre las categorías de las variables independientes), Wilcoxon (para determinar si existen diferencias entre el rango de dos muestras), U de Mann-Whitney (en caso de encontrar una significancia asintótica relevante) y el coeficiente Tau de Kendall (medida de correlación para variables ordinales). El análisis de los datos nos ayudó a tomar decisiones de cara a crear un guion para los grupos de discusión. Para realizarlo se cumplieron criterios de rigor como la confirmabilidad, fiabilidad, aplicabilidad, neutralidad y credibilidad, y se contó con la ayuda de otros diez profesores expertos, que ayudaron a reformular algunas preguntas y añadir tres nuevas.

Una vez realizado el grupo de discusión, analizamos los datos con el programa informático Atlas.ti, lo que nos sirvió para realizar el siguiente guion, el de las entrevistas a editores expertos de la Wikipedia. Una vez más se contó con profesores universitarios para validar el guion, cumpliendo además los mismos criterios de rigor científico explicados para el guion de los grupos de discusión. Se reformularon cinco preguntas y se procedió a realizar las entrevistas, que se analizaron con Atlas.ti.

Tabla 1. Resumen de preguntas de las herramientas de obtención de información

\begin{tabular}{l|c|c|c} 
Dimensión & Ítems cuestionario & Ítems grupo & Ítems entrevista \\
\hline Datos personales & 9 & 3 & 4 \\
\hline Parte general de la Web 2.0 & 36 & 11 & 1 \\
\hline Consulta de la Wikipedia & 27 & 7 & 5 \\
\hline Edición de la Wikipedia & 5 & $\mathbf{2 3}$ & $\mathbf{2 2}$ \\
\hline Total & $\mathbf{9 1}$ & & 2 \\
\hline
\end{tabular}

Fuente: elaboración propia (2020).

\section{Participantes}

En el año en que se realizó esta investigación fueron 156.986 los alumnos que se matricularon en todas las universidades de España (públicas o privadas) en estudios de Educación. Para calcular el número de cuestionarios necesarios para considerar los resultados como válidos, tomamos un nivel de confianza del $95 \%$ y un error del 3\%. Teniendo en cuenta los citados valores, el número de cues- 
18 tionarios necesarios era de 1.060. Una vez recibidas todas las respuestas de los alumnos universitarios españoles, se tuvieron que validar, ya que algunas fueron contestadas por profesores y por alumnos en universidades extranjeras, y se obtuvieron finalmente 1.173 .

Correspondieron a mujeres 959 respuestas (81\%), debido a que el número de matriculadas es muy superior en estudios de Educación. Por su parte, 775 fueron respondidas por alumnos menores de 23 años (66\%) y 272 por alumnos de 24 a 30 años, debido a que los alumnos universitarios matriculados suelen ser menores de 30 años. Respecto a los estudios que cursaban, 427 alumnos (37\%) cursaban el Grado en Magisterio en Educación Primaria y 275 (23\%) el Grado en Magisterio en Educación Infantil. En relación con el año en el que estudiaban, las respuestas fueron muy repartidas entre los cuatro cursos de grado. La mayoría de los alumnos que cumplimentaron el cuestionario afirmaron tener un conocimiento medio (48\%) o avanzado (34\%) en informática, mientras que la mayoría navegaban entre 1 y 2 horas al día (37\%) o entre 2 y 4 horas (37\%).

Tras analizar todas las respuestas obtenidas se creó el guion del grupo de discusión, que fue desarrollado con estudiantes de máster en alguna especialidad de Educación en la Universidad de Barcelona. El grupo de alumnos acudió voluntariamente a la reunión y lo formaron cinco mujeres y un hombre, con edades comprendidas entre los 22 y los 39 años.

Siguiendo el modelo secuencial, una vez analizados los datos del grupo de discusión se realizó el guion de las entrevistas a los editores expertos de la Wikipedia. Respondieron a las preguntas un total de nueve usuarios, previamente seleccionados entre los editores de la Wikipedia con más de 30.000 ediciones, realizadas tras varios años en el proyecto. Fueron entrevistados un total de cuatro mujeres y cinco hombres, con edades comprendidas entre los 21 y los 77 años, y con diferentes niveles de estudios, desde personas con el graduado escolar hasta graduados universitarios.

\section{RESULTADOS}

En primer lugar, analizamos el conocimiento que admiten tener los alumnos sobre la Wikipedia. El resultado fue un valor de 2,88, lo que indica un conocimiento medio-alto. Analizando los resultados de este ítem, tenemos los siguientes datos descriptivos: 
Tabla 2. Datos descriptivos del ítem Nivel de conocimiento de la Wikipedia respecto de las variables independientes

\begin{tabular}{|c|c|c|c|c|c|c|}
\hline & & Recuento & Mínimo & Máximo & Media & Mediana \\
\hline \multirow{2}{*}{ Sexo } & Masculino & 210 & 1,00 & 4,00 & 2,95 & 3,00 \\
\hline & Femenino & 942 & 1,00 & 4,00 & 2,87 & 3,00 \\
\hline \multirow{5}{*}{ Edad } & Menos de 23 años & 763 & 1,00 & 4,00 & 2,92 & 3,00 \\
\hline & Entre 24 y 30 años & 269 & 1,00 & 4,00 & 2,86 & 3,00 \\
\hline & Entre 31 y 40 años & 87 & 1,00 & 4,00 & 2,74 & 3,00 \\
\hline & Entre 41 y 50 años & 26 & 1,00 & 4,00 & 2,65 & 3,00 \\
\hline & Más de 51 años & 7 & 2,00 & 4,00 & 2,86 & 3,00 \\
\hline \multirow{6}{*}{$\begin{array}{l}\text { ¿Qué estudios } \\
\text { realizas? }\end{array}$} & $\begin{array}{l}\text { Grado en Magisterio en } \\
\text { Educación Primaria }\end{array}$ & 417 & 1,00 & 4,00 & 2,87 & 3,00 \\
\hline & $\begin{array}{l}\text { Grado en Magisterio en } \\
\text { Educación Infantil }\end{array}$ & 271 & 1,00 & 4,00 & 2,82 & 3,00 \\
\hline & Grado en Pedagogía & 154 & 1,00 & 4,00 & 2,92 & 3,00 \\
\hline & Grado en Educación Social & 130 & 1,00 & 4,00 & 2,94 & 3,00 \\
\hline & $\begin{array}{l}\text { Grado en Magisterio en } \\
\text { Infantil y Primaria }\end{array}$ & 45 & 1,00 & 4,00 & 2,89 & 3,00 \\
\hline & Máster & 135 & 1,00 & 4,00 & 2,97 & 3,00 \\
\hline \multirow{4}{*}{$\begin{array}{l}\text { Curso más alto } \\
\text { de grado }\end{array}$} & Primer curso de grado & 262 & 1,00 & 4,00 & 2,85 & 3,00 \\
\hline & Segundo curso de grado & 209 & 1,00 & 4,00 & 2,94 & 3,00 \\
\hline & Tercer curso de grado & 215 & 1,00 & 4,00 & 2,86 & 3,00 \\
\hline & Cuarto curso de grado & 308 & 1,00 & 4,00 & 2,85 & 3,00 \\
\hline \multirow{4}{*}{ Titulación } & Diplomatura o grado & 920 & 1,00 & 4,00 & 2,88 & 3,00 \\
\hline & Máster a la vez que grado & 93 & 1,00 & 4,00 & 2,85 & 3,00 \\
\hline & Máster & 135 & 1,00 & 4,00 & 2,97 & 3,00 \\
\hline & Doctorado & 4 & 1,00 & 3,00 & 2,00 & 2,00 \\
\hline \multirow{5}{*}{$\begin{array}{l}\text { ¿Cuál es tu nota } \\
\text { media en la } \\
\text { carrera? }\end{array}$} & Entre 5 y 5,9 & 22 & 2,00 & 4,00 & 2,68 & 3,00 \\
\hline & Entre 6 y 6,9 & 199 & 1,00 & 4,00 & 2,92 & 3,00 \\
\hline & Entre 7 y 7,9 & 435 & 1,00 & 4,00 & 2,90 & 3,00 \\
\hline & Entre 8 y 8,9 & 229 & 1,00 & 4,00 & 2,87 & 3,00 \\
\hline & Más de 9 & 30 & 1,00 & 4,00 & 2,87 & 3,00 \\
\hline
\end{tabular}




\begin{tabular}{|c|c|c|c|c|c|c|c|}
\hline 20 & & & Recuento & Mínimo & Máximo & Media & Mediana \\
\hline \multirow{13}{*}{ 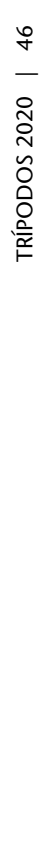 } & \multirow{5}{*}{$\begin{array}{l}\text { ¿Posees otra } \\
\text { titulación? }\end{array}$} & Ninguna & 697 & 1,00 & 4,00 & 2,93 & 3,00 \\
\hline & & $\begin{array}{l}\text { Ciclo Formativo de Grado } \\
\text { Superior }\end{array}$ & 279 & 1,00 & 4,00 & 2,76 & 3,00 \\
\hline & & $\begin{array}{l}\text { Ingeniería Técnica o } \\
\text { Diplomatura ( } 3 \text { años) }\end{array}$ & 80 & 1,00 & 4,00 & 2,91 & 3,00 \\
\hline & & $\begin{array}{l}\text { Ingeniería o Licenciatura } \\
\text { (5 años) }\end{array}$ & 64 & 2,00 & 4,00 & 2,91 & 3,00 \\
\hline & & Posgrado & 32 & 2,00 & 4,00 & 2,88 & 3,00 \\
\hline & \multirow{4}{*}{$\begin{array}{l}\text { ¿Qué nivel de } \\
\text { conocimiento } \\
\text { informático } \\
\text { posees? }\end{array}$} & Nivel básico & 175 & 1,00 & 4,00 & 2,62 & 3,00 \\
\hline & & Nivel medio & 558 & 1,00 & 4,00 & 2,84 & 3,00 \\
\hline & & Nivel avanzado & 396 & 1,00 & 4,00 & 3,04 & 3,00 \\
\hline & & Nivel experto & 18 & 2,00 & 4,00 & 3,50 & 4,00 \\
\hline & \multirow{4}{*}{$\begin{array}{l}\text { ¿Cuántas horas } \\
\text { al día navegas } \\
\text { en internet? }\end{array}$} & Menos de 1 hora & 79 & 1,00 & 4,00 & 2,66 & 3,00 \\
\hline & & Entre 1 y 2 horas & 425 & 1,00 & 4,00 & 2,85 & 3,00 \\
\hline & & Entre 2 y 4 horas & 430 & 1,00 & 4,00 & 2,88 & 3,00 \\
\hline & & Más de 4 horas & 211 & 1,00 & 4,00 & 3,03 & 3,00 \\
\hline
\end{tabular}

Fuente: elaboración propia (2020).

Las diferencias entre las categorías son muy leves, variando entre ,2 y ,3 puntos, exceptuando los dos últimos ítems, donde la diferencia llega hasta ,88 entre los alumnos con nivel informático básico y los expertos, y ,37 entre los que dedican menos de una hora y los que navegan más de cuatro. Para completar esta información obtuvimos los porcentajes de cada categoría de respuestas, donde se observó que los mayores porcentajes se dieron en la respuesta de "bastante conocimiento", que sobrepasaba el 50\% en la mayoría de los casos. Únicamente el 1,74\% de los encuestados admitió tener un conocimiento nulo de la Wikipedia, aunque solo el $17,10 \%$ afirma que la conoce mucho. Cabe destacar que los alumnos en general creen tener conocimientos sobre la Wikipedia, siendo casi el 75\% los que admiten tener bastante conocimiento.

Seguidamente utilizamos la prueba de Kruskal-Wallis, que reveló que existe significancia asintótica en la posesión de otra titulación, el nivel de conocimiento informático y las horas al día que navegan los alumnos universitarios españoles en internet. Tras obtener estos datos se realizó la prueba U de Mann-Whitney para los tres ítems que indicaban anteriormente una significancia asintótica relevante, además de los ítems del sexo y de la titulación de postgrado. 
Tabla 3. Prueba de U de Mann-Whitney aplicada al ítem Nivel de conocimiento de la Wikipedia en función de las variables independientes

\begin{tabular}{|c|c|c|c|c|c|c|}
\hline & Valor 1 & Valor 2 & $\begin{array}{l}\text { U Mann- } \\
\text { Whitney }\end{array}$ & $\begin{array}{c}\text { W } \\
\text { Wilcoxon }\end{array}$ & Z & $\begin{array}{c}\text { Sig. } \\
\text { Asintót }\end{array}$ \\
\hline $\begin{array}{l}\text { B1.06 } \\
\text { Titulación de } \\
\text { postgrado }\end{array}$ & Máster & Doctorado & 98.5 & 108.5 & -2.468 & ,014 \\
\hline $\begin{array}{l}\text { B1.08 ¿Posees } \\
\text { otra titulación? }\end{array}$ & Ninguna & C.F.G.S. & 83589 & 122649 & -3.826 & ,000 \\
\hline \multirow{6}{*}{$\begin{array}{l}\text { B1.09 Nivel } \\
\text { conocimiento } \\
\text { informático }\end{array}$} & \multirow{3}{*}{ Nivel básico } & Nivel medio & 40813.5 & 56213.5 & -3.716 & , 000 \\
\hline & & Nivel avanzado & 24469.5 & 39869.5 & -6.129 & ,000 \\
\hline & & Nivel experto & 614 & 16014 & -4.650 & ,000 \\
\hline & \multirow{2}{*}{ Nivel medio } & Nivel avanzado & 94224 & 250185 & -4.352 &, 000 \\
\hline & & Nivel experto & 2493 & 158454 & -4.158 &, 000 \\
\hline & Nivel avanzado & Nivel experto & 2312.5 & 80918.5 & -2.761 & ,006 \\
\hline \multirow{5}{*}{$\begin{array}{l}\text { B2.01 Horas al } \\
\text { día en internet }\end{array}$} & \multirow{3}{*}{$\begin{array}{l}\text { Menos de } 1 \\
\text { hora }\end{array}$} & Entre 1 y 2 horas & 14328 & 17488 & -2.323 & ,020 \\
\hline & & Entre 2 y 4 horas & 14218.5 & 17378.5 & -2.548 & 011 \\
\hline & & Más de 4 horas & 6064 & 9224 & -3.945 & ,000 \\
\hline & $\begin{array}{l}\text { Entre } 1 \text { y } 2 \\
\text { horas }\end{array}$ & Más de 4 horas & 38710.5 & 129235.5 & -3.155 & ,002 \\
\hline & $\begin{array}{l}\text { Entre } 2 \text { y } 4 \\
\text { horas }\end{array}$ & Más de 4 horas & 40256.5 & 132921.5 & -2.576 & 010 \\
\hline
\end{tabular}

Fuente: elaboración propia (2020).

Tras analizar los datos de la tabla superior observamos que a medida que los alumnos dedican más horas a conectarse a internet y afirman tener un mayor conocimiento informático, también lo tienen respecto a la Wikipedia.

Posteriormente, analizamos el coeficiente Tau de Kendall, que nos indica la relación existente entre dos variables. Se analizaron las variables sobre las horas de conexión y del conocimiento informático, y se observó que, en algunos casos, a medida que los alumnos navegan más horas en internet $(\tau b=, 099)$ y que tienen un conocimiento informático mayor $(\tau b=, 196)$ también aumenta su nivel de conocimiento en la Wikipedia.

Una vez que supimos el conocimiento que afirmaban tener sobre la Wikipedia, quisimos conocer si los alumnos sabían que podían editar en la Wikipedia, ya que esto indicaría mínimamente el conocimiento que tienen sobre su funcionamiento. Una gran mayoría de los alumnos $(92,2 \%)$ sabía que se podía editar en la Wikipedia, a pesar de que el 5\% todavía no lo sabe y la utiliza bastante o mucho. Es menor el número de alumnos que no lo saben y la utilizan poco o 
nada, exactamente el 2,96\%. Los alumnos de la Universidad de Barcelona sabían que podían editar en la Wikipedia, pero tenían dudas al respecto de los procesos de edición, ya que no sabían si había máquinas o usuarios que impedían las ediciones con contenidos erróneos.

A continuación, quisimos saber el número de estudiantes universitarios españoles que editan en la Wikipedia. La mayoría no lo hace, lo que se constata con una media de 1,14, valor que se encuentra cerca de la respuesta "Nunca". También se les cuestionó sobre el uso que le dan a la Wikipedia y la frecuencia con la que editan en esta enciclopedia en línea:

Tabla 4. Uso que le dan a la Wikipedia y colaboración en ella

\begin{tabular}{l|l|c|c|c|c}
\multicolumn{2}{c}{} & \multicolumn{2}{c}{ ¿Has colaborado alguna vez en la Wikipedia? } \\
\cline { 3 - 6 } & & Nunca & Ocasionalmente & Frecuentemente & $\begin{array}{c}\text { Cada vez que } \\
\text { tengo tiempo } \\
\text { libre }\end{array}$ \\
\hline \multirow{3}{*}{$\begin{array}{l}\text { ¿Qué uso le das a } \\
\text { la Wikipedia? }\end{array}$} & Nada & 18 & 2 & 0 & 0 \\
\cline { 2 - 6 } & Poco & 370 & 37 & 0 & 0 \\
\cline { 2 - 6 } & Bastante & 425 & 53 & 1 & 0 \\
\cline { 2 - 6 } & Mucho & 109 & 31 & 2 & 1 \\
\hline
\end{tabular}

Fuente: elaboración propia (2020).

Respecto a la cantidad de alumnos que han editado en alguna ocasión, solo han sido 126 , lo que supone el $12 \%$ del total, mientras que el $0,38 \%$ lo ha hecho frecuentemente o muy a menudo. Asimismo, se quiso conocer la cantidad de ediciones que habían realizado los estudiantes de la Universidad de Barcelona, pero tan solo uno de ellos admitió haber editado en una ocasión, lo que confirma así la baja tasa de editores entre los alumnos (É1: Me fastidió mucho no haber encontrado los niveles educativos ni en catalán ni en castellano de una forma correcta $y$ actualizada, entonces lo edité. Una colaboración solo), aunque la opinión general de todos ellos fue que era muy positivo que la gente editase artículos en ella (É1: Yo creo que es bueno, que está bien).

En cuanto a las razones por las que los estudiantes universitarios no editan en la Wikipedia, el 55\% de las mujeres contestó "No creo que pudiese aportar nada"; mientras que en el caso de los hombres el porcentaje que afirmó lo mismo fue menor, $44,5 \%$. La mitad de los universitarios españoles opinó que no podría aportar nada, mientras que el 38,4\% de los hombres y el 27,8\% de las mujeres afirmaron que se informarían sobre cómo editar. Vistos los resultados obtenidos de ambos sexos, se realizó el test de Kruskall-Wallis para observar si existía una diferencia significativa entre hombres y mujeres, y se confirmó finalmente la diferencia $(\mathrm{p}=, 017)$.

Los alumnos de la Universidad de Barcelona indicaron que el motivo por el cual no editaban no era la falta de interés, sino que consideraban que no podrían 
aportar nada más a lo que ya existe (E2: De lo que busco no sé más información), así como otros argumentos como la falta de seguridad y su excesivo perfeccionismo (E6: Yo en mi caso no me atrevería, o sea es como no atreverse, porque siempre piensas que algo lo tienes que conocer más. No estoy del todo segura porque aún tengo que continuar contrastando aquello, o parte del aprendizaje), o el hecho de no haber encontrado el momento adecuado para ello (E1: Yo no he editado más veces porque tampoco he encontrado el momento; E4: Dices, ya lo pondré otro día, y no me costaría nada).

También se citó en dos ocasiones el desconocimiento técnico (E6: El conocimiento técnico de saber poner aquello alli; E5: Tienes que tener unos conocimientos sobre ello). A este respecto, en el cuestionario se obtuvo un 1,36 de media en la afirmación "Mis profesores nos han explicado cómo usarla y para qué", cerca de la respuesta "Completamente en desacuerdo". Esto nos indica que la mayoría de los alumnos no han recibido ningún tipo de formación sobre la Wikipedia y que además sus profesores no les han indicado que debe consultarse con precaución. El 77,8\% está en desacuerdo con la afirmación que indica que los profesores les dicen que la usen y referencien con precaución, mientras que el 91,4\% está en desacuerdo con la afirmación que indica que sus profesores les han explicado cómo usarla. En el grupo de discusión, a ninguno le habían explicado su funcionamiento, en ocasiones por falta de conocimientos tecnológicos del profesorado (E1: Si pasan de los 50 años ya no, no usan Wikipedia; E2: El problema está en que nosotros vamos ahí y sabemos utilizar Wikipedia, sabemos lo que es, pero a ver, los profesores no).

Para los editores expertos, las principales causas por las que los alumnos no editan en la Wikipedia son la falta de tiempo (W2: Se necesita tiempo; W3: Proponer a alguien que prolongue su vida laboral con tareas voluntarias sobre su propio trabajo no parece lo mejor; W7: El hecho de dedicar tiempo y esfuerzo a algo que ni da dinero ni méritos es un hándicap), el miedo (W4: Por miedo), el desconocimiento (W9: Desconocimiento sobre el proyecto y su posibilidad de editar libremente; W5: Cierta fracción de la población universitaria más bien tecnófoba) y, por los comentarios de otras personas, que no tienen una buena opinión de ella (W1: Falta de fiabilidad de la que es acusada; W5: Postura general de la población respecto a Wikipedia; W4: Visión demonizada de Wikipedia que casi por ósmosis se transmite al resto de la población universitaria). Sin embargo, afirmaron que cada vez se realizan más actividades con alumnos y que la mayoría de los editores fueron universitarios.

Los alumnos universitarios están de acuerdo en que los editores cedan su conocimiento al proyecto, con una media de 3,04, porque ellos son los beneficiados de ese crecimiento de contenidos. Para analizar los resultados con mayor profundidad, a continuación, mostramos el recuento de ambas variables relacionadas entre sí. 
Tabla 5. Recuento de los alumnos que han colaborado en la Wikipedia y la opinión que tienen respecto a la cesión del conocimiento

\begin{tabular}{|c|c|c|c|c|c|}
\hline & \multicolumn{4}{|c|}{ ¿Has colaborado alguna vez en ella? } \\
\hline & & Nunca & Ocasionalmente & Frecuentemente & $\begin{array}{c}\text { Cada vez } \\
\text { que tengo } \\
\text { un momento } \\
\text { libre }\end{array}$ \\
\hline \multirow{4}{*}{$\begin{array}{l}\text { ¿Qué opinas sobre } \\
\text { que los editores de } \\
\text { la Wikipedia cedan } \\
\text { su conocimiento } \\
\text { libremente a la } \\
\text { comunidad de } \\
\text { usuarios? }\end{array}$} & $\begin{array}{l}\text { En ningún caso } \\
\text { debería hacerse, } \\
\text { yo cobro por mis } \\
\text { conocimientos }\end{array}$ & 8 & 0 & 0 & 0 \\
\hline & $\begin{array}{l}\text { Me parece } \\
\text { interesante pero } \\
\text { no es mi prioridad }\end{array}$ & 368 & 32 & 0 & 0 \\
\hline & $\begin{array}{l}\text { Yo puedo editar } \\
\text { contenidos sobre } \\
\text { mis hobbies, pero } \\
\text { no sobre mi área } \\
\text { profesional }\end{array}$ & 43 & 6 & 0 & 0 \\
\hline & $\begin{array}{l}\text { Bien, todos } \\
\text { deberíamos } \\
\text { hacerlo }\end{array}$ & 417 & 85 & 3 & 1 \\
\hline
\end{tabular}

Fuente: elaboración propia (2020).

Aquellos alumnos que opinan que ellos cobran por sus conocimientos nunca han colaborado con la Wikipedia. También observamos que la mayoría de los alumnos que colabora ocasionalmente piensa que todos deberíamos ceder nuestro conocimiento $(69,11 \%)$; mientras que otra gran parte de ellos $(26,02 \%)$ piensa que es interesante editar en la Wikipedia, aunque no es su prioridad. Del total, 417 alumnos opinan que todos deberíamos ceder nuestro conocimiento libremente a la Wikipedia, pero nunca han colaborado, lo que supone el 82,41\%. Del resto, el 16,8\% (85 alumnos) afirma que edita ocasionalmente; mientras que solo cuatro estudiantes escriben en la Wikipedia con asiduidad, a pesar del número tan alto que ha contestado que todos deberíamos editar (506 alumnos).

Después de muchos años editando, todos los editores de la Wikipedia creen que están capacitados para editar, pero añaden que cualquiera con sentido común puede escribir en la Wikipedia (W1: Todos, o casi todos, estamos capacitados para ello. Apliquemos el sentido común y la prudencia a la hora de editar; W3: En términos generales, lo están. Capacidad, tienen; W4: Entiendo que la mayor parte del alumnado sí lo está, por la formación que han ido adquiriendo en su vida académica; W5: Cualquier persona está capacitada para editar en Wikipedia con una cierta formación; W7: Para editar Wikipedia solo hace falta tiempo, ganas y conexión a internet; W9: El proyecto se basa en la premisa de que cualquier persona con unos mínimos co- 
nocimientos informáticos y culturales esté capacitada para editarlo). El hecho de que la Wikipedia no sea fuente primaria hace que los editores no sean los creadores de información, sino que son los que leen la información en otras fuentes, escriben sobre ello en la Wikipedia e indican de donde han obtenido los datos (W6: No hace falta ser un escritor, pues no es necesario crear el artículo, solo argumentarlo con referencias en fuentes fiables; W8: Todos podemos incluir el último resultado de un partido de rugby, basta enlazar con el periódico digital en el que se ha publicado la noticia de ese encuentro, no hace falta ser periodista para ello ni hacer una mayor profundidad).

\section{DISCUSIÓN Y CONCLUSIONES}

Los resultados que dieron respuesta al primer objetivo de la investigación —constatar el conocimiento que tienen los alumnos universitarios sobre la Wikipedia- fueron medio-altos, tal y como indican la media del ítem "¿Qué nivel de conocimiento tienes de la Wikipedia?" $(2,88)$ y el hecho de que solo el $1,74 \%$ afirmó tener un conocimiento nulo de la Wikipedia. Observando las variables independientes analizadas, constatamos datos similares, en la mayor parte de las categorías, que llegan en algunas a un nivel de muy alto. Además, se observó que cuanto mayor es el nivel informático que poseen los alumnos, más conocimiento afirman tener sobre la Wikipedia. Asimismo, observamos el mismo efecto en los alumnos que dedican más horas a navegar por internet. Cuantas más horas empleadas, mayor conocimiento afirman poseer.

Flanagin y Metzger (2011) comprobaron que, aunque la mayoría de los alumnos encuestados conocía la Wikipedia, el 22\% no sabía que todo el mundo podía participar. En nuestro caso, el 92,2\% respondió afirmativamente a esta pregunta, quizá debido a que en los últimos años los alumnos tienen mayor conocimiento del funcionamiento de la Wikipedia. Los estudiantes de la Universidad de Barcelona también sabían que se podía editar, pero tenían dudas sobre la facilidad de insertar información falsa, por lo que creemos que se deberían fomentar iniciativas desde los centros docentes para mejorar las competencias de la búsqueda de información, tal y como recomiendan Valverde-Crespo et al. (2019).

Para dar respuesta al segundo objetivo marcado, conocer si editan o han editado en alguna ocasión esta enciclopedia en línea, analizamos el ítem "¿Has colaborado alguna vez en ella?". Los resultados indicaron que solo 126 alumnos habían editado en alguna ocasión en la Wikipedia, lo que supone el 12\% del total, mientras que el 0,38\% lo ha hecho frecuentemente o muy a menudo. Dichos datos se encuentran en la línea de los apuntados por Kleimann et al. (2008). En su investigación, el 23\% de los alumnos había editado en alguna ocasión y el 1\%, frecuentemente. Esta baja tasa de editores también ha sido constatada en otras investigaciones (Brox, 2012; Sánchez, 2014).

Las razones que expusieron los estudiantes de la Universidad de Barcelona y los editores expertos para no editar en la Wikipedia fueron variadas. Principalmente manifestaron que no se veían capaces de aportar nada más a lo que ya existe, la falta de seguridad, su excesivo perfeccionismo, la mala reputación de 
la Wikipedia, la falta de tiempo, el hecho de no haber encontrado el momento adecuado para ello y el desconocimiento técnico. Sobre esta última cuestión, los alumnos fueron muy críticos con los profesores, indicando que no les explicaban cómo utilizarla, para qué y los momentos en que podía ser útil. Los resultados obtenidos a este respecto coinciden con las conclusiones de Cuquet y García-San Pedro (2019), pero fueron más negativos que los resultados obtenidos por Sahut et al. (2015).

Brox (2012) constató igualmente que existía un desconocimiento técnico por parte de los usuarios a la hora de editar en la Wikipedia, pero al contrario de lo obtenido en ese estudio, muchos estudiantes afirmaron que estarían dispuestos a contribuir en un futuro. A pesar de la negatividad de los estudiantes en algunas respuestas al respecto de su capacidad y conocimientos, los editores expertos opinaron que cualquiera con sentido común puede escribir en la Wikipedia, teniendo en cuenta que deben leer la información en otras fuentes, y escribir sobre ello en la Wikipedia, indicando la fuente de información.

Los alumnos universitarios están de acuerdo en que los editores cedan su conocimiento al proyecto, y el 52,5\% piensa que todos deberíamos ceder nuestro conocimiento a esta enciclopedia (el 69,1\% de los que colaboran ocasionalmente piensa así). A pesar de estos datos, el 82,4\% de los que opinaron así nunca ha colaborado con la Wikipedia. En total solo cuatro estudiantes escriben en la Wikipedia con asiduidad. Por su parte, el 41,5\% piensa que es interesante editar en la Wikipedia, pero no es su prioridad.

En general, se constató la poca participación de los alumnos universitarios en la Wikipedia, debido a una gran variedad de razones, entre las que se encuentra su percepción de encontrarse poco preparados e inseguros para hacerlo, en parte porque no han recibido una formación adecuada. Creemos que el estudio supone una contribución importante a la difusión de la situación de los estudiantes en instituciones de educación superior, a pesar de que el estudio está limitado por el tipo de alumnos utilizados, exclusivamente de Educación.

Ángel Obregón-Sierra (angel.obregon@ui1. es). Profesor colaborador en la Universidad Isabel I y en la Universidad Oberta de Catalunya, es doctor en Educación y graduado en Informática e Historia. Miembro de Wikimedia

Natalia González-Fernández (natalia.gonzalez@unican.es). Profesora titular de la Universidad de Cantabria en el Área MIDE en la Facultad de Educación. Trabaja desde hace 23 años en investigación, docencia, gestión y transfe-
España, editor de la Wikipedia desde hace más de 13 años e investigador sobre el uso que se da a esta enciclopedia en Educación. Ha publicado varios artículos sobre sus investigaciones en la Wikipedia.

rencia de conocimiento, centrándose en metodologías activo-reflexivas, competencia mediática y evaluación formativa. Ha publicado más de 60 artículos y más de 25 trabajos en libros. Coordina el Grupo I+D+i de la UC "MILET". 


\section{Bibliografía}

Brox, H. (2012). "The Elephant in the Room: A Place for Wikipedia in Higher Education?" Nordlit, 30, pp. 143-155.

Cuquet, M. y García-San Pedro, M. J. (2019). "Percepciones y uso de la Wikipedia en alumnos de educación secundaria". Education in the Knowledge Society, 20(0): 15. DOI: $<10.14201 /$ eks2019_20_a8>.

Ebner, M.; Zechner, J. y Holzinger, A. (2010). "Why Is Wikipedia so Successful? Experiences in Establishing the Principles in Higher Education'. I-KNOW '06. Graz. Disponible en: <https://www.researchgate.net/ publication/228905272>. Consultado el $24 \mathrm{de}$ marzo de 2020.

Endrizzi, L. (2006). L'édition de référence libre et collaborative : le cas de Wikipedia. Équipe LIRE - Université Lyon 2. Disponible en: $<$ https://archivesic.ccsd.cnrs.fr/sic_00473760/ document>. Consultado el 24 de marzo de 2020.

Fessakis, G. y Zoumpatianou, M. (2012). "Wikipedia Uses in Learning Design: A Literature Review". Themes in Science and Technology Education, 5(1/2), pp. 97-106.

Flanagin, A. J. y Metzger, M. J. (2011). "From Encycopaedia Britannica to Wikipedia. Generational Differences in the Perceived Credibility of Online Encyclopedia Information". Information, Communication \& Society, 14(3), pp. 355-374. DOI: $<10.1080 / 1369118 X .2010 .542823>$.

Forte, A. y Bruckman, A. (2010). "Writing, Citing, and Participatory Media: Wikis As Learning Environments in the High School Classroom". International Journal of Learning and Media, 1(4), pp. 23-44.

Gilles, M. (2015). Wikipédia, une encyclopédie collaborative en quête de crédibilité: le référencement en questions (Tesis doctoral). Universidad de Toulouse. Disponible en: $\quad<$ https://tel.archives-ouvertes.fr/tel01257207/document>. Consultado el 26 de marzo de 2020.
Gómez, M. A. [et al.] (2014). “Ordenadores en el aula: ¿estamos preparados los profesores?". Enseñanza de las Ciencias, pp. 239-250.

Head, A. J. y Eisenberg, M. B. (2009). "How Today's College Students Use Wikipedia for Course-Related Research". First Monday. Peer-reviewed Journal on the Internet, 15(3). Disponible en: <http://firstmonday.org/article/ view/2830/2476>. Consultado el 26 de marzo de 2020 .

Kleimann, B.; Özkilic, M. y Göcks, M. (2008). "Studieren im Web 2.0. Studienbezogene Web- und E-Learning-Dienste". HISBUSKurzinformation, 21. Hanover. Disponible en: $<$ https://hisbus.his.de/hisbus/docs/hisbus21. pdf $>$. Consultado el 24 de marzo de 2020.

Knight, Ch. y Pryke, S. (2012). "Wikipedia and the University, a Case Study". Teaching in Higher Education, 17(6), pp. 649-659.

Meishar-Tal, H. (2015). "Teachers' Use of Wikipedia with Their Students". Australian Journal of Teacher Education, 40(12). DOI: $<10.14221$ /ajte.2015v40n12.9>.

Menchen-Trevino, E. y Hargittai, E. (2011). "Young Adults' Credibility Assessment of Wikipedia Information". Communication \& Society, 14(1), pp. 24-51. DOI: $<10.1080 / 13691181003695173>$.

Meseguer-Artola, A. (2014). "Aprendiendo mediante la comparación con Wikipedia: su importancia en el aprendizaje de los estudiantes". RUSC. Universities and Knowledge Society Journal, 11(2), pp. 57-69. DOI: <10.7238/rusc. v11i2.2042>.

Monereo, C. (2009). "Competencia digital: para qué, quién, dónde y cómo debe enseñarse". Aula de Innovación Educativa, 181, pp. 9-12.

Obregón, A. (2019). "Evolution of Students' Editions". Wikimedia Education Conference. San Sebastián. Disponible en: <https:// cutt.ly/etE5iro $>$. Consultado el 26 de marzo de 2020. 
Peters, D. (2012). "Wikipedia Education Program Case Studies". Fundación Wikimedia. Disponible en: <https://cutt.ly/7tE5yVG>. Consultado el 24 de marzo de 2020.

Queiroz de, J-A. (21 de marzo de 2014). "Ecrire pour Wikipedia, ça s'apprend maintenant à la fac". Le Figaro. Disponible en: $<$ https://cutt.ly/atE76uO >. Consultado el 23 de marzo de 2020.

Sahut, G. y Chabriac, K. (2015). "Quand des lycéens contribuent à Wikipédia: quelles évolutions de leurs connaissances informationnelles?" En: Loicq, M. y Rio, F. (dir.) Les jeunes: Acteurs des médias. Participation et accompagnement. Centre d'Études sur les Jeunes et les Médias, pp. 108-118.

Sahut, G. [et al.] (2015). "Qu'apprennent les jeunes usagers á propos de Wikipédia?" En: Barbe, L.; Merzeau, L. y Schafer, V. Wikipédia objet scientifique non identifié. Presses universitaires de Paris Ouest, pp. 149-161. Disponible en: <http://archivesic.ccsd.cnrs.fr/ sic_01360441/document>. Consultado el 27 de marzo de 2020.
Sánchez, L. (2014). Utilización de Wikipedia como recurso docente en la enseñanza superior (Tesis doctoral). Universidad de Salamanca. DOI: $<10.14201 /$ gredos.125932>.

Saorín, T. (2012). Wikipedia de la A la W. Barcelona: UOC.

Saorín, T.; Haro, V. de y Pastor, J. A. (2011). "Posibilidades de Wikipedia en la docencia universitaria: Elaboración colaborativa de conocimiento". Ibersid: Revista de sistemas de información y documentación, 5, pp. 89-97.

Valverde-Crespo, D.; González-Sánchez, J. y Pro-Bueno, A. (2019). "Wikipedia en la Universidad: ¿Cómo la utilizan los estudiantes de $1^{\underline{o}}$ curso de Grado de titulaciones del área de Ciencias Experimentales? Un estudio sobre sus percepciones". Revista Eureka sobre Enseñanza y Divulgación de las Ciencias 16(3), pp. 3101. DOI: <10.25267/Rev_Eureka_ensen_divulg_ cienc.2019.v16.i3.3101>.

Zachte, E. (2019). Wikipedia Statistics Spanish. Disponible en: <https://stats.wikimedia. org/EN/TablesWikipediaES.htm>. Consultado el 28 de marzo de 2020 . 